\title{
Optimization of Coating Thickness of Conducting Material on Base Fiber to make it as a Radar Reflector
}

\author{
Vivek Bajpai, Verandra Kumar, Kishan Lal Gadri, Ajit Kumar Singh, Niyaz Mohammad, Kavita Chouhan, Alok \\ Basita, Prashant Vasistha*, and Ravindra Kumar \\ Defence Laboratory, DRDO, Jodhpur, 342011, INDIA \\ *Corresponding Author: pvasistha@yahoo.com
}

\begin{abstract}
Chaff is one of the most extensively used passive electronic countermeasure to disrupt radar tracking and are designed to cover wide frequency range from 2-18 GHz. There are various factors to improve backscattered RCS of the chaff cloud at higher frequencies. In this paper, the effect of coating thickness of conducting material of electrically conducting fibre (which is used as chaff) on its backscattered RCS has been studied. The relationship between fiber coating thickness of conducting material and its backscattered RCS were obtained by performing simulations in Ansys HFSS with dipole length of $50 \mathrm{~mm}$ and $15 \mathrm{~mm}$ and later validating the same with the measured results. It is observed that with an increase in coating thickness of conducting material of the fibre there is increase in its backscattered RCS. When coating thickness of conducting metal becomes greater than the skin depth of the conducting metal, the increase in observed RCS with increase in coating thickness is marginal. Thus, further increase in coating thickness greater than the skin depth of the conducting metal will not improve the backscattered RCS of the fiber significantly but it will add weight penalty for the fiber.
\end{abstract}

Keyword: - Backscattered RCS, electrically conducting fiber, HFSS simulations, skin depth, anechoic chamber

\section{Introduction}

First used during World War II to confuse the enemy tracking radar systems, chaff still remains one of the most cost effective and constantly used passive electronic countermeasure till date. Chaff consists of large number of thin, highly conducting wires, cut to form dipoles of varying lengths that are resonant at specific frequencies of microwave radiations [1], [2].

Many factors like chaff cut lengths, number of dipoles, mutual coupling, screening effects, polarization of EM waves, chaff ejection method etc. affects the RCS behavior of chaff payload [3]. Thus, for an effective chaff payload design, effects thin dipole having optimized coating is to be analyzed keeping over all weight and its persistence in air in consideration.

RCS simulation using Ansys HFSS which is followed by RCS measurement of chaff fibers with varying coating thickness of conducting material in anechoic chamber at 2$18 \mathrm{GHz}$. A good agreement in the simulation and measurement results is observed.

Radar Cross Section is a ratio of backscattered power per steradian in the direction of the radar from the target to the power density incident on the target. Mathematically, RCS can be represented by [4], [5].

$$
\sigma=\lim _{R \rightarrow \infty} 4 \pi R^{2}\left|\frac{S r}{s t}\right|
$$

Here, $R$ is the range, $S r$ is the reflected power per steradian and $S t$ is the transmitted power density.

A lot of work has been done on scattering of electromagnetic waves from a metallic wire [6]-[10]. RCS simulation and measurement of microwave dipoles has been reported in [11]. Realization of low RCS and wide beam radiating element for $\mathrm{Ku}$-band phased array has been reported in [12]. Effect of surface roughness on backscattered RCS of chaff fiber has been studied in [13]. In this paper, for optimization of backscattered RCS of chaff fibre, effect of coating thickness of conducting metal (copper) over a base fibre has been studied. Conducting metal chosen for coating is copper as it has excellent conductivity and small skin depth.

Skin depth is that distance below the surface of a conductor where the current density has diminished to (1/e) of its value at the surface. 14]:

$$
\operatorname{Skin} \operatorname{Depth}(\delta)=\sqrt{\frac{\rho}{\pi f \mu_{r} \mu_{o}}}
$$

Where $r=$ resistivity of the material (ohm/meter), this is inverse of conductivity

$f=$ frequency of the current

$\mu=\mu_{r} \mu_{o}$ permeability of the material, where $\mu_{o}=4 \pi 10^{-7}$ $(\mathrm{H} / \mathrm{m})$

Terminal velocity of any particle is the steady speed achieved by the particle freely falling through a gas or liquid.

Mathematically, terminal velocity of a particle is given as [2]:

$$
\begin{aligned}
& v_{t}=\frac{g * \Delta \rho * c^{2}}{6 * \mu}\left[\left\{\ln \left(\frac{l}{c}\right)-\frac{3}{2}\right\} \sin ^{2} \theta+\left\{\ln \left(\frac{l}{c}\right)-\frac{1}{2}\right\}\right] \\
& v_{\mathrm{t}}=\text { terminal velocity } \\
& \mu=\text { viscosity of the fluid }=1.983 \times 10^{-5} \mathrm{Kg} / \mathrm{m} . \mathrm{s} \text { for air } \\
& \mathrm{g}=\text { acceleration due to gravity } \\
& \Delta \rho=\text { density difference between fiber and air } \\
& \mathrm{c}=\text { radius of fiber } \\
& \mathrm{l}=\text { length of fiber }
\end{aligned}
$$

In this paper, measurements are performed using a conducting metal (copper) coated over a low density base fibers. The fiber with different metal coating over base fibers 
are taken for the study of effect of coating thickness of conducting metal (copper) on backscattered RCS of chaff fibers. The mean diameters of the base fibre used for chaff fiber preparation is 6.5 micron. On these base fibers, conducting metal coating of thickness ranging from $0.5 \mu \mathrm{m}$ to $4 \mu \mathrm{m}$ is performed to prepare chaff fibers which gives diameter range of chaff fibers from 7 to 10.5 microns.

\section{Simulated results}

\subsection{Simulated RCS data}

Simulations are performed using Ansys HFSS [15], [16]. HFSS-IE solver is used to simulate RCS of chaff dipoles. Two different cut lengths of chaff dipole viz $50 \mathrm{~mm}$ and 15 $\mathrm{mm}$ are considered with varying diameter of chaff fiber viz. $7.5,8.5,9.5,10.5$, and 11.5 micron.

Simulation of rectangular strips is much easier as compared to cylindrical fibers, since meshing of 2D planar object is simple as compared to meshing of $3 \mathrm{D}$ curved surface in the order of micron range. Since the radius of chaff fiber is in $\sim 5$ micron therefore to mesh the curved surface of chaff fiber in the order of sub-micron reduces the size of mesh element and increasing the number of meshing elements. Increase in meshing element increases the time for simulation. We have performed a simulation in which we compared the simulated RCS results of rectangular strip and a cylindrical fiber and it is observed that there is not much significant difference in backscattered RCS of the rectangular strips and cylindrical fibers of same width (diameter) thus cylindrical fibers are models as rectangular fiber in HFSS in order to avoid time consuming meshing in simulations. Figure 1 and Figure 2 show the simulated backscattered RCS for $50 \mathrm{~mm}$ and $15 \mathrm{~mm}$ long chaff dipole array with varying diameters.

\subsection{Effect of weight on terminal velocity of chaff}

The chaff fiber is metal coated base fibers. Generally, the density of base fiber is typically around $\sim 1900 \mathrm{~kg} / \mathrm{m}^{3}$ while that of conducting metal (Copper) is nearly 4 times that of a base fiber, thus with small increase in coating thickness of conducting metal over base fiber there will be huge increase in the net weight of the fiber. Figure 3 demonstrate that weight of the chaff fiber increases drastically with an increase in metal coating thickness.

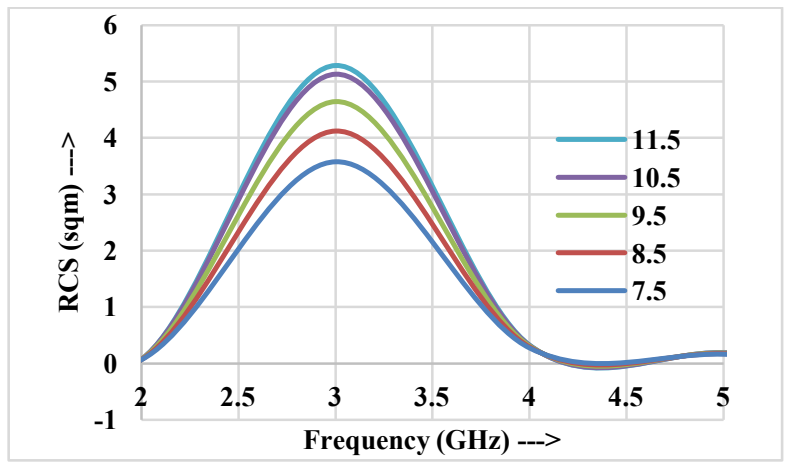

Fig. 1. Simulated backscattered RCS of $50 \mathrm{~mm}$ chaff fibers. Units of legends is in $\mu \mathrm{m}$ (micron)

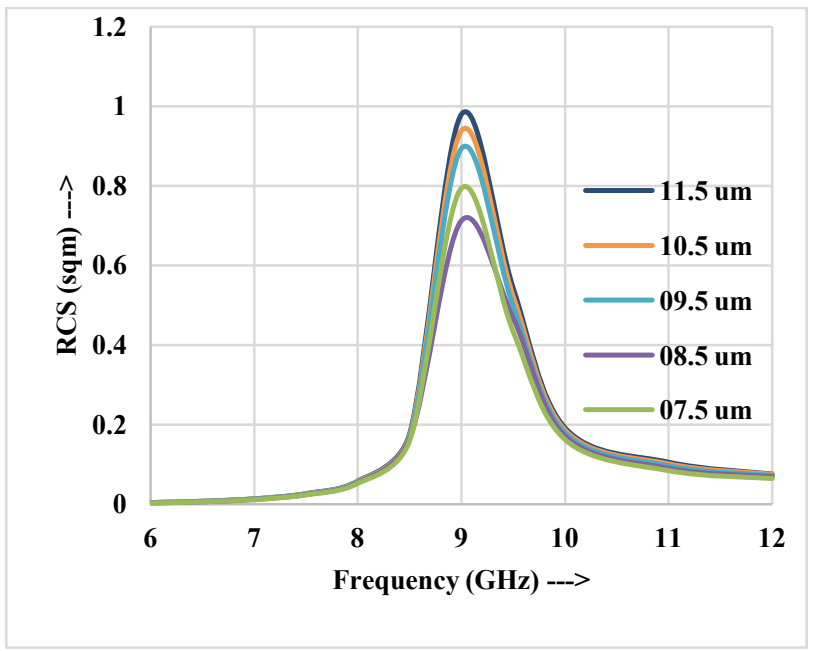

Fig. 2. Simulated backscattered RCS of $15 \mathrm{~mm}$ chaff fibers. units of legends is in $\mu \mathrm{m}$ (micron)

\section{Relation between coating thickness vs Weight of metal coated carbon fiber of length $50 \mathrm{~mm}$ and 15 mm}

Density of Carbon Fiber $=1.93 \mathrm{~g} / \mathrm{cm} 3$

Density of a heavy metal $=8.96 \mathrm{~g} / \mathrm{cm} 3$

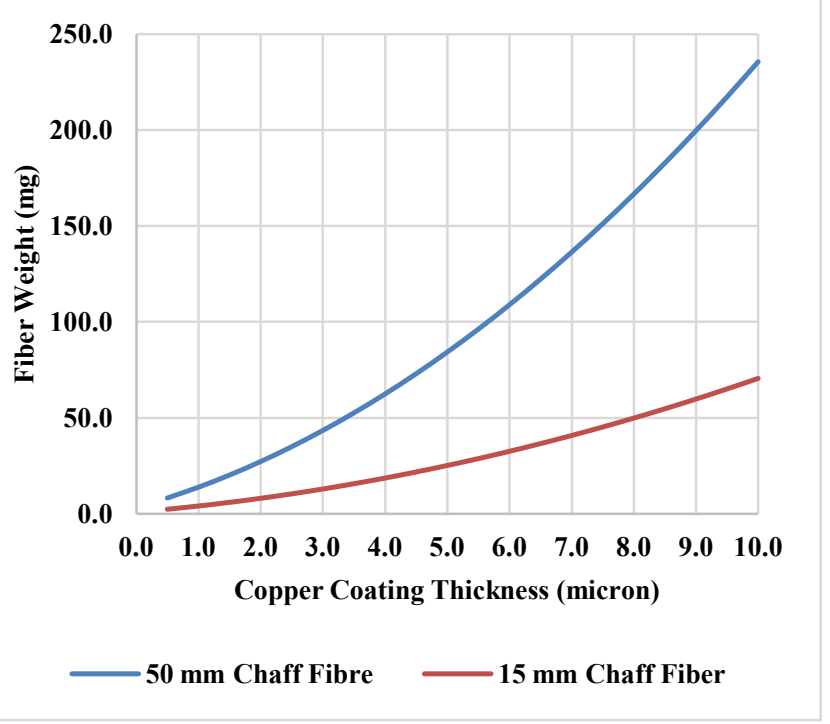

Fig. 3. Theoretical relation between metal coating thickness $(\mu \mathrm{m})$ and total weight of $50 \mathrm{~mm}$ and $15 \mathrm{~mm}$ Chaff fibers.

The effect of an increase in weight of the chaff fiber on its terminal velocity is also estimated. Since terminal velocity of chaff fibre is an important parameter as it decide the settling time of chaff cloud. Ideally, chaff cloud should remain suspended in air for longer time and for that one need terminal velocity of the chaff fiber to be low. Chaff fiber with higher terminal velocity will tend to settle faster as compared to the lower terminal velocity. In Figure 4, relationship between terminal velocity and chaff fiber equivalent weight and orientation is shown. It can be observed from Figure 4 that with higher weight of the chaff fiber, the terminal velocity increases thus resulting in faster settling of the chaff cloud. Thus one need to minimize chaff fiber weight as much as possible for longer settling time. 


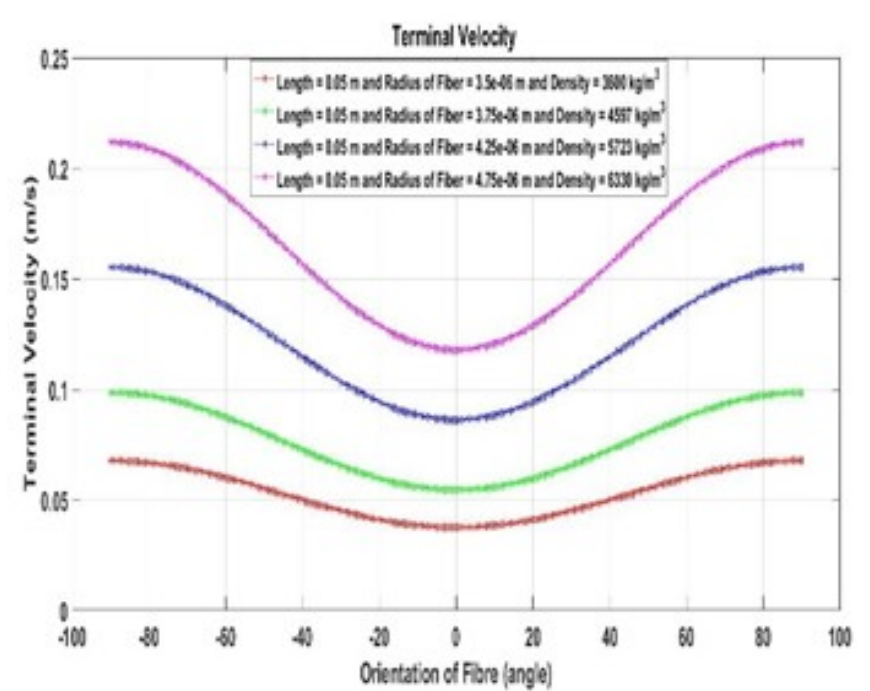

Fig. 4. Terminal velocity of metal coated carbon with different copper coating thickness over carbon fiber

\section{Experimental setups and measurements}

\subsection{Fiber diameter and coating thickness measurement}

Direct measurements of electrically conducting fiber diameters are performed using Optical Microscope with resolution of 0.37 micron at $100 \mathrm{x}$ magnification [17]. Image of a chaff fiber taken from optical microscope is shown in Figure 5 .

\subsection{Preparation of dipole array}

For a study of backscattered RCS behavior of different chaff dipoles of given samples, dipole arrays of fixed arrangements (horizontal and vertical separation) of predefined dipole cut length $(50 \mathrm{~mm}$ and $15 \mathrm{~mm})$ are prepared to evaluate effects of different coating thickness of conducting material over base fiber on its backscattered RCS. Figure 6 shows the schematic of dipole array design used for the experiments. Cut length $(\lambda / / 2)$ of the chaff dipoles used are $50 \mathrm{~mm}$ and $15 \mathrm{~mm}$ in order to cover two . The horizontal spacing between each dipole is $\lambda / 2$ and vertical spacing is $\lambda / 4$. Figure 7 shows the prepared test coupons (Dipole Array) for measurements.

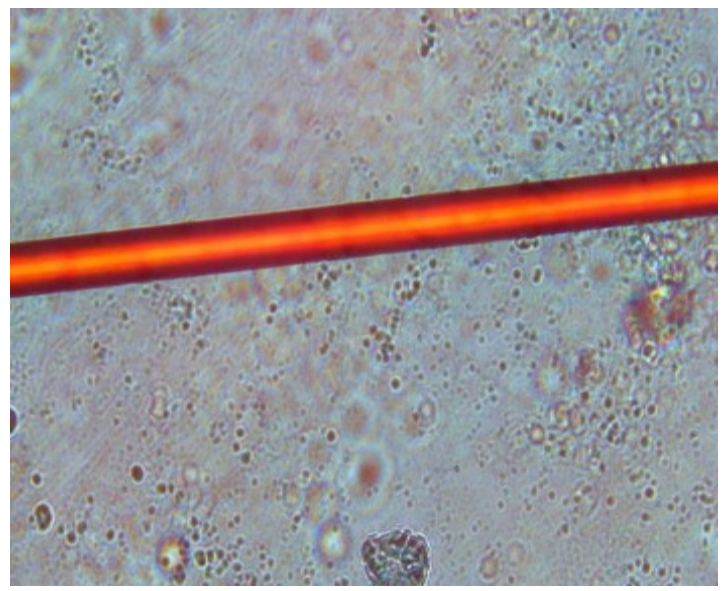

Fig. 5. A typical metal (copper) coated chaff fiber

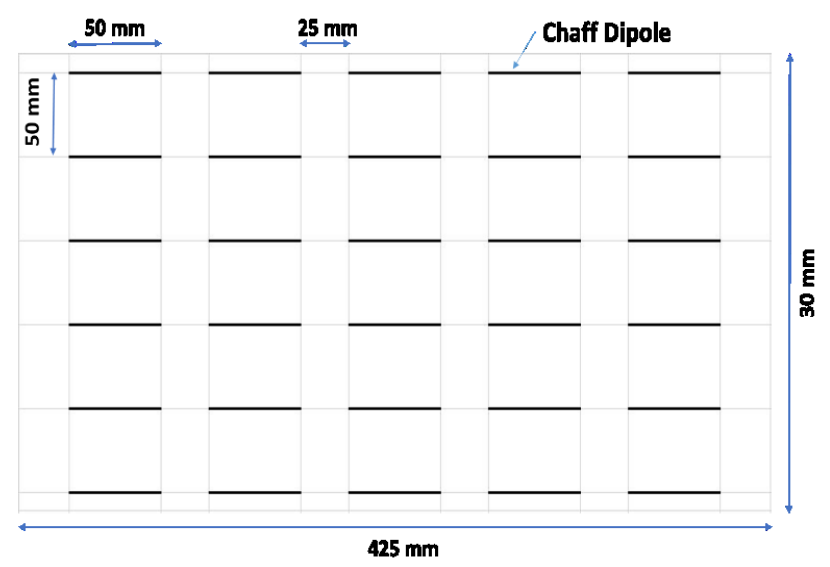

Fig. 6. Schematic of chaff dipole array design in HFSS

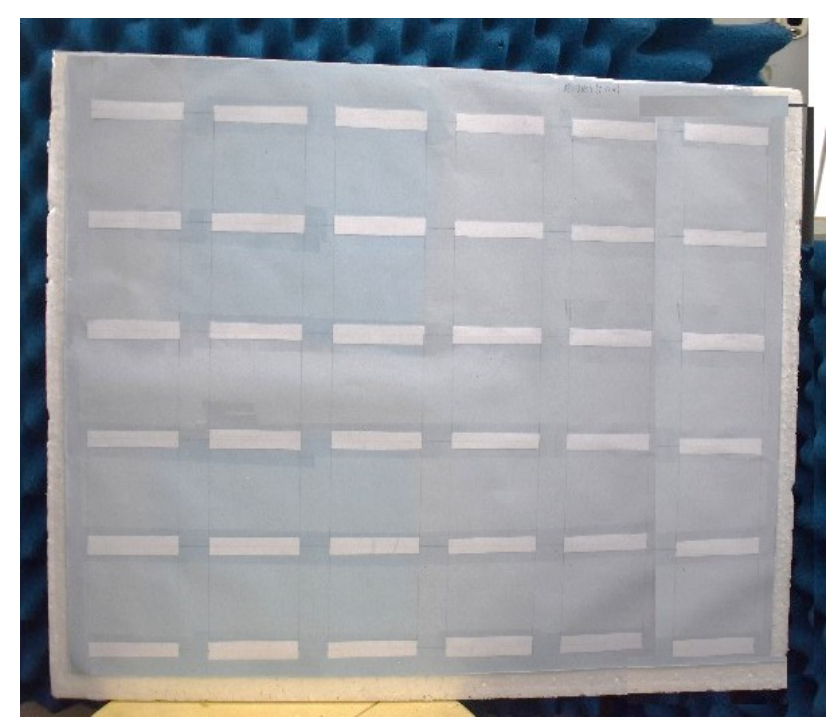

(a)

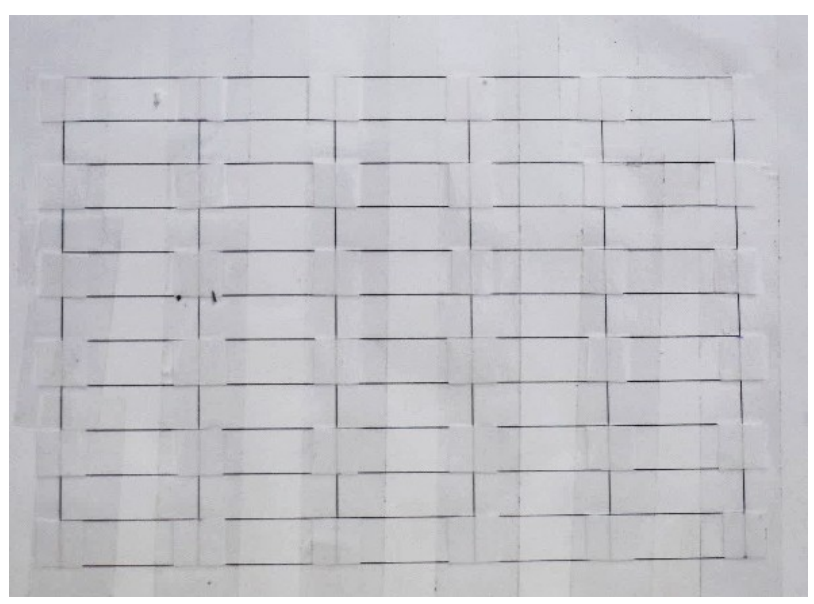

(b)

Fig. 7. Array of metal coated base fiber used for RCS measurement in Anechoic Chamber (a) $50 \mathrm{~mm}$ length (b) $15 \mathrm{~mm}$ length

\subsection{RCS measurement setup}

In the present study, time domain techniques are used for backscattered RCS measurements. In Time domain (TD) technique, ultra-wide band pulse of $\sim 30$ picosecond time 
duration is used as test signals and measurements are carried out over the frequency range of 2-18 GHz.

Defence Laboratory Jodhpur (DLJ) has established a Time Domain RCS Measurement facility, a unique of its own kind in the country, having Laboratory Time Domain RCS Measurement instrumentation and associated accessories comprising of Differential Pulse generator, Real Time Oscilloscope, Digital Sampling Oscilloscope, time domain antennas, computer controller, positioner etc. to measure $2-20 \mathrm{GHz}$. The facility is capable of doing monostatic RCS measurement from 2-20 GHz using time domain instrumentation which enables RCS measurement \& ISAR imaging of UUT (Maximum weight of UUT - $100 \mathrm{Kg}$ ). It has facility for automatic calibration with a standard reference target. The system also has filters to eliminate GSM frequencies to reduce errors.

Figure 8 shows the experimental setup for TD static RCS measurements of chaff dipole arrays. Since it was not feasible to perform RCS measurement of bloomed metallic fiber cloud in anechoic chamber, therefore a different approach for test coupon fabrication has been thought off. For this, metallic fiber are pasted on microwave transparent 2D thermocoal sheet and measurements are performed.

\subsection{Measured data}

Measured data are shown in from Fig. 9Figure 9 to 12 for various coating thickness for chaff fibers of $50 \mathrm{~mm}$ and $15 \mathrm{~mm}$ cut lengths. It can be observed that initially with increase in conducting metal coating over base fiber increases backscattered RCS and slowly it get saturate as the coating thickness of conducting metal comes in the range of 1 to 2 times of skin depth of the conducting metal at the operating frequency. Figure 1 and Figure 2 show the monostatic RCS of the chaff fiber at resonant frequencies with increasing coating thickness of a conducting metal on a base fiber. It can be easily observed that backscattered RCS increases with increase in coating thickness of the conducting metal till $\sim 2$ micron (in the range of 1 to 2 times of the skin depth of the conducting material at operating frequency) then it get saturated.



Fig. 8. Indoor Backscattered RCS measurement set up in Anechoic Chamber

\section{Measured RCS of $50 \mathrm{~mm}$ chaff fibres with varying coating thickness (micron)}

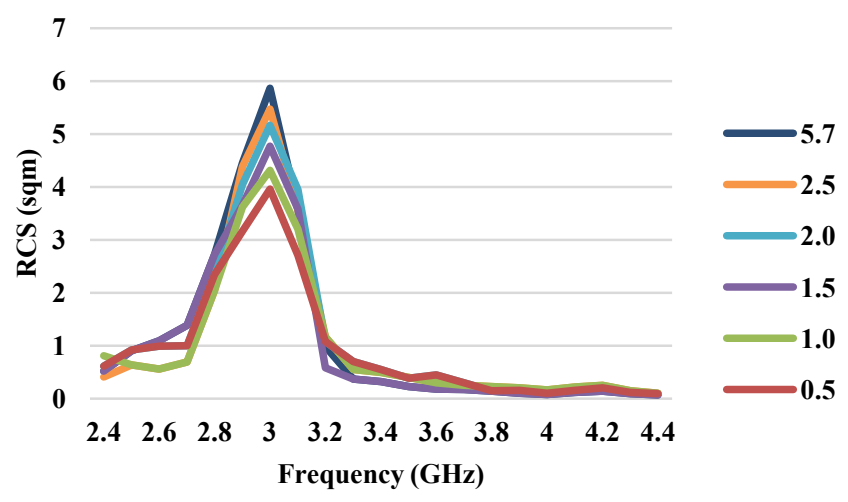

Fig. 9. Measured backscattered RCS of $50 \mathrm{~mm}$ Chaff Fibers. Units of legends (coating thickness) is in $\mu \mathrm{m}$ (micron)

\section{Measured RCS of $15 \mathrm{~mm}$ Chaff Fiber with varying coating thickness (micron)}

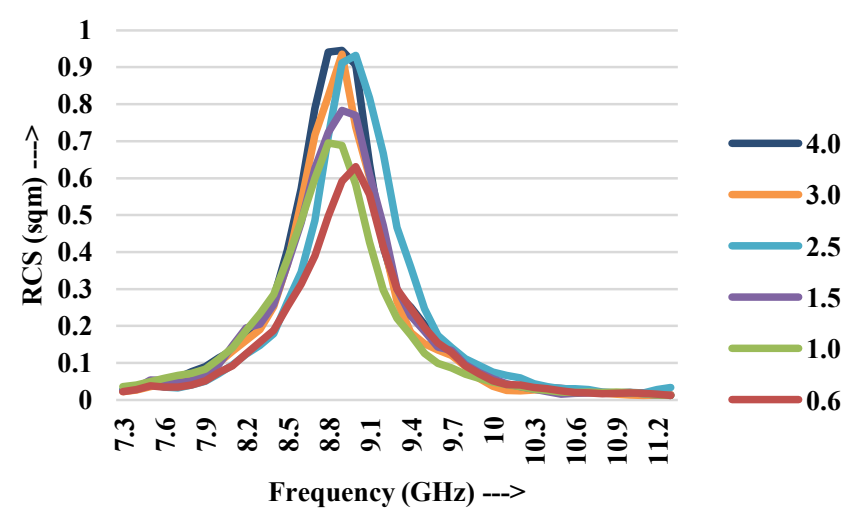

Fig. 10. Measured Backscattered RCS of $15 \mathrm{~mm}$ Chaff Fibers. Units of legends (coating thickness) is in $\mu \mathrm{m}$ (micron)

\section{Measured RCS vs Metal Coating Thickness for 50mm Chaff Fiber @ Resonant Frequency of $3 \mathrm{GHz}$}

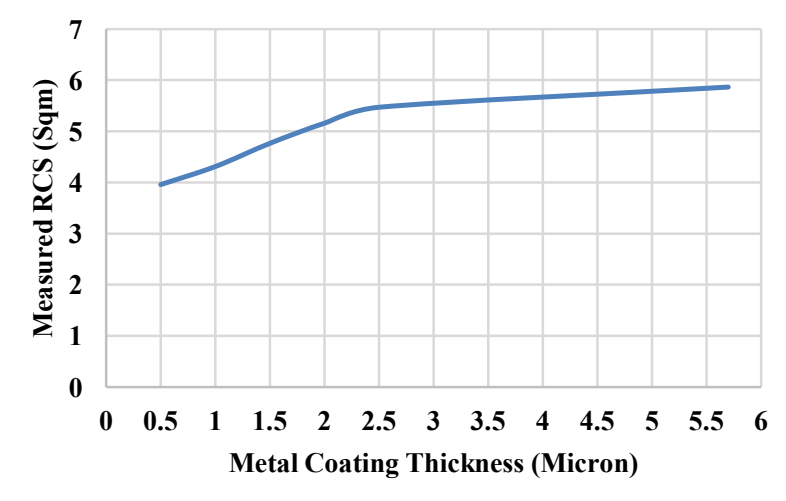

Fig. 11. Relationship observed between measured RCS and conducting metal coating thickness of $50 \mathrm{~mm}$ Chaff fiber at $3 \mathrm{GHz}$ 


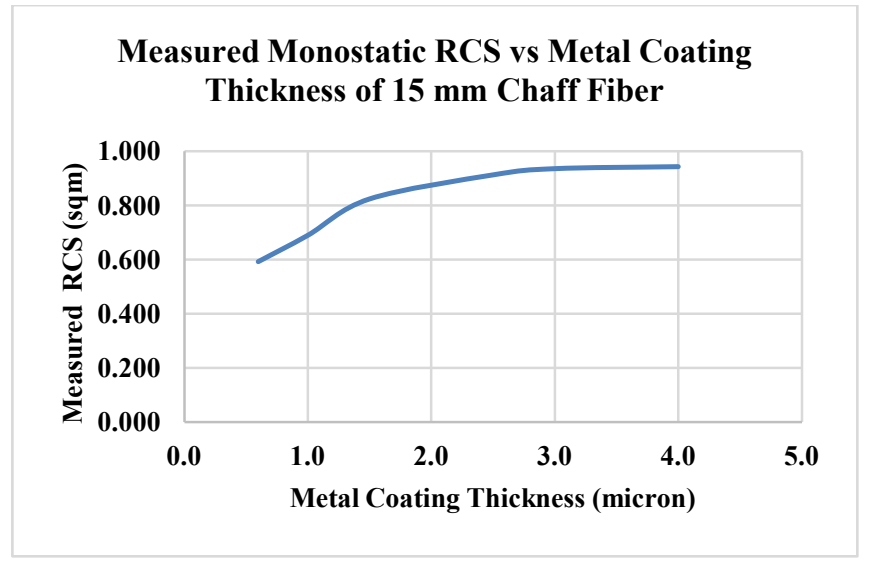

Fig. 12. Relationship observed between measured RCS and conducting metal coating thickness of $15 \mathrm{~mm}$ Chaff fiber at $9 \mathrm{GHz}$

\section{CORRELATION BETWEEN SIMULATED AND \\ MEASURED RCS OBSERVED AT RESONANCE FREQUENCY WITH VARIOUS COATING THICKNESS}

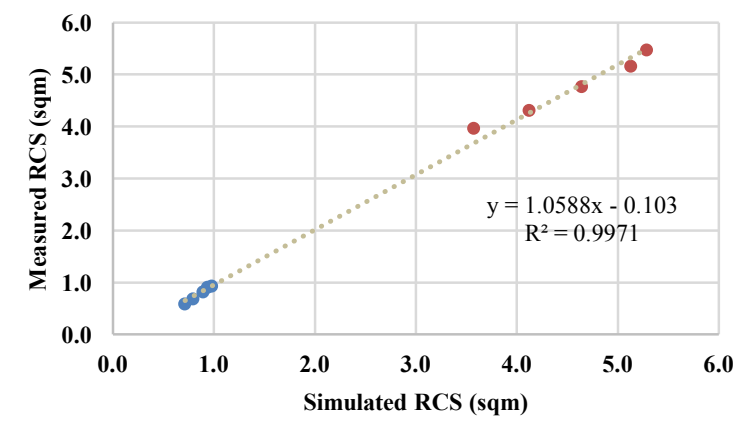

Fig. 13 Correlation between simulated and measured RCS at resonance frequency with varying coating thickness (Blue for 15 $\mathrm{mm}$ and Red for $50 \mathrm{~mm}$ Chaff fibres)

\section{Results and discussion}

It is observed from both simulated and measured data as shown in Figure 11 and 12 that with an increase in fiber coating thickness of conducting material there is an increase in its backscattered RCS. This trend goes on until one reaches coating thickness of the conducting metal in the range 1 to 2 times of skin depth (SD) of the conducting material at operating frequency. With further increase in coating thickness (greater than twice of the skin depth of the material), increase in its backscattered RCS response becomes less prominent. Thus, from the results it can be concluded that the most optimized coating thickness of the conducting metal over any light weight base fiber should be in the range 1 to 2 times of Skin Depth of the material at the operating frequency. With further increase in coating thickness of conducting metal greater than twice the skin depth will not give any significant improvement in backscattered RCS but its weight penalty for the fiber will start dominating as the density of conducting metal is generally large compare to density of base fiber. In addition, this increase in weight will increase the terminal velocity of the chaff fibres resulting in faster settling of the chaff fibers.
From Figure 13, a good correlation between simulated and measured data at resonance frequencies is observed for both $50 \mathrm{~mm}$ and $15 \mathrm{~mm}$ chaff fibres for various coating thickness of the conducting metal. The coefficient of determination (R2), which is the measure of linear correlation between two data sets (simulated and measured) is 0.9971 as shown in Figure 13, indicating a good correlation between simulated and measured data.

The chaff does not resonate at exact resonant frequency which it was designed for, the reason supporting the above claim is that the dipole thickness should be infinitesimally small to resonate at theoretical frequency [1] which is beyond fabrication point of view, so a minimum acceptable value of dipole thickness is taken to compensate for the shift in peak. Also, if the thickness is further reduced the peak shift more toward the resonant frequency. The curve sharpness as well as resonant length of dipole depends on the "Aspect Ratio", which is a ratio of length to diameter (1/d) of the fibre. The same can be observed form simulated and measured data sets. Shifting of resonance frequency is more pronounced in $15 \mathrm{~mm}$ chaff than the $50 \mathrm{~mm}$ chaff as diameters of the chaff fibres is same but the length of fibres is comparatively less for $15 \mathrm{~mm}$ chaff fibres making lower aspect ratio ( $1 / \mathrm{d})$ for $15 \mathrm{~mm}$ chaff fibres as compared to 50 $\mathrm{mm}$ chaff fibres.

Backscattered RCS of chaff cloud is directly proportional to the number of the chaff dipole elements present in the chaff payload [1]. Analytically, the maximum RCS is given by [18]

$$
\sigma(\max )=\frac{G^{2} \lambda^{2}}{\pi}
$$

where $\mathrm{G}$ is the gain and $\lambda$ is the wavelength.

The maximum gain in far field for half wavelength antenna is given by

$$
\Sigma_{\max }=0.856 \lambda^{2}
$$

For random orientation of dipole and averaging over $4 \pi$ for $\mathrm{N}$ elements,

$$
\sigma=0.172 * \lambda^{2}
$$

From equation (6), RCS of dipoles $(\sigma)$ is directly proportional to the number of dipoles $(\mathrm{N})$.

In practical applications of chaff, the chaff payload weight is specifically given and remains constant for a given design. In a given specified payload weight (W), the number of dipoles $(\mathrm{N})$ are given by-

$$
N=\frac{w}{v * \rho}
$$

$v=$ Volume of a fiber $=\pi r^{2} l$,

Here, $\mathrm{r}=$ radius of the fiber and $l=$ length of the fiber, and $\rho=$ Equivalent density of the fiber.

Let's consider two fibers $\left(f_{1}\right.$ and $\left.f_{2}\right)$ with fiber radius $r_{1}$ and $r_{2}$ and equivalent density $\rho_{1}$ and $\rho_{2}$ respectively. The radius of base fiber is $r_{\mathrm{b}}$. Then, the number of dipoles in a specified weight (W) of chaff payload are-

For a chaff payload consisting of fibre $\left(f_{1}\right)$ only

$$
N_{1}=\frac{W}{v_{1} * \rho_{1}}
$$

and 
For a chaff payload consisting of fibre $\left(f_{2}\right)$ only

$$
N_{2}=\frac{w}{v_{2} * \rho_{2}}
$$

Where, $v_{1}=\pi\left(r_{1}\right)^{2} 1$,

$$
v_{2}=\pi\left(r_{2}\right)^{2} 1
$$

For any given fiber with radius $r_{f}$ and length $l$ and equivalent density of $\rho_{e q}$, let base fiber density is $\rho_{b}$ and conducting metal density is $\rho_{c}$, then the volume of fiber is given by-

$$
\begin{array}{r}
\pi r_{f}^{2} l=\frac{\left[\rho_{b} * r_{b}^{2}+\rho_{c} *\left(r_{f}^{2}-r_{b}^{2}\right)\right] \pi l}{\rho_{e q}} \\
\text { or, } \quad \rho_{e q}=\frac{\rho_{b^{*}} r_{b}^{2}+\rho_{c^{*}}\left(r_{f}^{2}-r_{b}^{2}\right)}{r_{f}^{2}}
\end{array}
$$

Putting equation 10 and 11 in equation 8 and 9 the equivalent densities of two fibres $f_{1}$ and $f_{2}$ can be computed as-

$$
\begin{aligned}
& \rho_{1}=\frac{\rho_{b} * r_{b}^{2}+\rho_{c} *\left(r_{1}^{2}-r_{b}^{2}\right)}{r_{1}^{2}}, \\
& \rho_{2}=\frac{\rho_{b} * r_{b}^{2}+\rho_{c} *\left(r_{2}^{2}-r_{b}^{2}\right)}{r_{2}^{2}}
\end{aligned}
$$

For same cut length $(l)$, from equation 8 and 9 we get,

$$
\frac{N_{1}}{N_{2}}=\frac{r_{2}^{2} \rho_{2}}{r_{1}^{2} \rho_{1}}
$$

Putting values of $\rho_{1} r_{1}^{2}$ and $\rho_{2} r_{2}^{2}$ from equation 12 and 13 in equation 14-

$$
\begin{aligned}
& \frac{N_{1}}{N_{2}}=\frac{\rho_{b} * r_{b}^{2}+\rho_{c} *\left(r_{1}^{2}-r_{b}^{2}\right)}{\rho_{b} * r_{b}^{2}+\rho_{c} *\left(r_{2}^{2}-r_{b}^{2}\right)} \\
& \frac{N_{1}}{N_{2}}=\frac{\left(\rho_{b}-\rho_{c}\right) * r_{b}^{2}+\rho_{c} * r_{2}^{2}}{\left(\rho_{b}-\rho_{c}\right) * r_{b}^{2}+\rho_{c} * r_{1}^{2}}
\end{aligned}
$$

From equation (16), it can be observed that as radius of the fiber increases (due to increase in coating thickness) the number of dipoles in chaff payload within a specified weight reduces. As per equation (6), reduction in number of dipoles in a chaff payload reduces its backscattered RCS response. Thus coating thickness of the conducting metal over base fiber plays an important role in optimization of chaff payload optimization.

\section{Conclusion}

In the present paper, optimization of coating thickness of conducting material (copper) over a light weight base fiber for maximum backscattered RCS is done. It is observed from both simulation and measured results that with an increase in coating thickness of the electrically conducting metal over a base fiber, there is an increase in its corresponding backscattered RCS response. However, when the coating thickness of conducting material reaches in the range of its skin depth to twice of the skin depth of the material at operating frequency than further increase in coating thickness will not contribute much in backscattered RCS of the chaff fiber. Thus, one should not increase coating thickness of conducting material much greater than twice of the skin depth, since it will not prove to be efficient in terms of improving its backscattered RCS, instead it will start increasing weight penalty for fiber as conducting metal (copper) are generally having high density compared to the base fibers. Increase in weight of the chaff fiber increases the terminal velocity of the chaff fibers, which results in faster settling of the chaff cloud. Thus, from the study it is concluded that the best optimized coating thickness of conducting material (copper) over base fiber should be in the range of 1 to 2 times the skin depth of the conducting metal at operating frequency.

\section{Acknowledgement}

The kind support and patronage received from Dr. S.V. Kamat, Distinguished Scientist and Director General DRDO is highly acknowledged. The support received from the entire team from Defence Laboratory Jodhpur who have directly or indirectly contributed to carry out this research work is acknowledged.

\section{References}

[1] B. C. Butters, Chaff, IEE Procedings, vol. 129, no. 3, pp. $197-$ 201, June 1982.

[2] S. W. Marcus, The dynamics and radar cross section density of chaff clouds, IEEE Transaction on Aerospace and Electronic Systems, vol. 40, no. 1, January 2004.

[3] V. Kumar, A. K. Singh, P. Vasistha and R. Kumar, Dynamic RCS prediction of rapidly blooming chaff cloud and its validation using measurement on scaled down, International Journal of Advances in Microwave Technology, vol. 3, no. 4, pp. 170-175, 2018.

[4] E. F. Knott, Radar Cross Section Fundamentals, in Radar Cross Section Measurements, First ed., New York, SciTech, 1993, pp. 14-17.

[5] M. L. Skolnik, Radar Cross Section of Targets, in Introduction to RADAR Systems, Second ed., Tata McGraw Hill, 1981, pp. $33-45$.

[6] O. Einarsson and T. Plato, Electromagnetic scattering by a thin resistive wire, Electronic Letters, vol. 5, no. 25, pp. 1019-1025, Dec 1969. DOI: 10.1049/el:19690476

[7] J. Rheinstein, Scattering of short pulses of electromagnetic waves, Proceedings of the IEEE, vol. 53, no. 8, pp. 1069-1070, Aug 1965. .

[8] Z. Yinan, J. Ming, Q. Xiaolin and Z. Zhiquan, Study on scattering characteristics of Chaff for Radar, Proceedings Microwave and Millimeter Wave Technology, pp. 673-676, 2004.

[9] Z. Zhou and J. S. Tyo, Transient analysis of straight thin wire scatterer by multiresolution time domain integral equation method, IEEE Antenna and Propagation Society, vol. 3, pp. 575578, 2003.

[10] N. Kruger, Modelling the EM properties of dipole reflections with application to uniform chaff clouds, Matieland, 2009.

[11] V. Kumar, P. Vasistha and R. Kumar, RCS simulation and measurement studies of microwave dipoles for chaff applications, International Journal of Advances in Microwave Technology, vol. 4, no. 1, pp. 185-189, 2020.

[12] R. Kumar, P. K. Verma and M. V. Kartikeyan, Realization of low RCS and wide beam radiating element for Ku-band electronically steered phased array, International Journal of Advances in Microwave Technology, vol. 4, no. 2, pp. 194-197, 2020.

[13] K. L. Gadri, V. Kumar, A. K. Singh, A. Basita, P. Vasistha and R. Kumar, Analysis of effect of surface roughness of electrically conducting fiber on its backscattered RCS, International Journal of Advances in Microwave Technology, vol. 5, no. 4, pp. 237$241,2021$. 
[14] W. M. Middleton and M. E. V. Valkenburg, Skin depth, in reference data for engineers- Radio, Electronics, Computer, and Communications, Ninth ed., Newnes, 2002, pp. 6-1-6-22.

[15] Ansys Inc., Fundamentals of HFSS, in An Introduction to HFSS, Ansys Inc, 2020, pp. 1-12.

[16] Ansys Inc., Multi Frequency Adaptive Meshing, in An Introduction to Multi Frequency Adaptive Meshing in HFSS, Ansys, Inc., 2017, pp. 1-12.

[17] Olympus, Performing Measurements, in User Manual Olympus Stream Image Processing Software, Olympus, pp. 48-51.

[18] C. L. Mack, B. REIFFEN and J., RF characteristics of thin dipoles, Proceedings of the IEEE, vol. 52, no. 5, pp. 533-542, May 1964. DOI: 10.1109/PROC.1964.2994

\section{Biography of the authors}

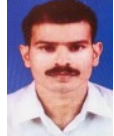

Vivek Bajpai: He did his MSc(Physics) from Madurai Kamaraj University, Madurai during 2007-2009. He joined DRDO at DL, Jodhpur in 2003and currently working as Technical Officer 'B'. He has experience of 17 years in the areas of development of water purification technologies and Modelling, Simulation and Measurements of electronic coutermeasure used by Armed forces

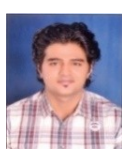

Verandra Kumar: He did his B Tech from MNIT, Jaipur in ECE during 2005-09. He joined DRDO in 2009 at DL, Jodhpur and currently working as Scientist D. His area of work includes RCS measurement and simulation of tagrets for stealth application, design of electronic countermeasure decoys as well as Dynamic RCS modelling, simulation and measurements of decoys currently being used by IAF and IN.

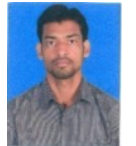

Kishan Lal Gadri: He did his B.Tech from Jaipur National University, Jaipur in ECE during 2007-2011. $\mathrm{He}$ has the experience of 08 years in the field of microwaves and its applications in remote sensing and modelling, simulation and RCS measurement of various electronic countermeasure systems at DRDO.

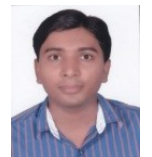

Ajit Kumar Singh: He joined DRDO in 2013 at DL, Jodhpur. He has the experience of 07 years in the area of design and development of software to simulate the parameters of electronic countermeasure and analysis of various chaff decoys used by IAF and IN.

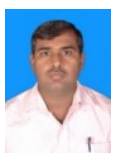

Niyaz Mohammad: He has joined DRDO in 2010 at DIC Panagarh (WB). He has 17 years experience in the field of Basic Electronics Engineering. He has 8 years of experience in the area of Strategic communication system(VSAT) and networking. He joined Defence Laboratory, Jodhpur in 2018. Presently he is working in the field of Static and Dynamic RCS Measurement of electronic counter measures.

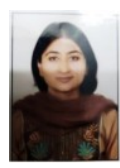

Kavita Chouhan: She did her MSc in Chemistry from JNVU, Jodhpur in 2000 and $\mathrm{PhD}$ in Chemical Kinetics from JNVU, Jodhpur in 2005. She joined DRDO in 2006 at HEMRL, Pune and worked on Composite propellant, Glycidyl azide based propellant. Currently, she is working as Scientist D in DRDO at DL Jodhpur on preparation of fine chaff fibres, their surface finishing, slip coating, and metal coating on fibres by electroless process.

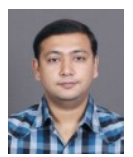

Alok Basita: He did his B.Tech from MNIT during 2001-2005. He joined DRDO in 2005 and currently working as scientist E at DL, Jodhpur. He has 15 years of experience in design of digital control systems for testing facility of various subsystems related to Gas Turbine Engine, electronic counter measure dispensing system, as well as RCS measurement studies on various targets and decoys suitable for electronic counter measure system.

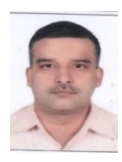

Prashant Vasistha: He did his PhD from Department of Electronics Engineering, IIT BHU. He joined DRDO in 1998 at DL, Jodhpur and currently working as Scientist G. He has been working in the area of Radar Cross Section (RCS) management and charectrization studies and RCS measurement technologies for combat systems and design and development of electronic countermeasure decoys. He has an experience of 24 years in the area of design and development of radar camouflage techniques and electronic counter measure decoys.

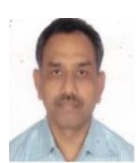

Ravindra Kumar: He did his BE from IIT Roorkee in 1983 and M Tech from IIT Mumbai in 1987. He joined DRDO in 1983 at DL, Jodhpur and currently working as Outsatnding scientist and Director of estalishment. He has an R\&D experience of 37 years in the area of desert warfare scenraio management, Phase change materials, water purifications systems and design, development and testing of electronic countermeasures. 